\title{
THE IMPACT OF A HIGHER EATING FREQUENCY ON THE QUALITY OF DIET AND NUTRITIONAL STATUS OF BREAST CANCER WOMEN UNDERGOING CHEMOTHERAPY
}

Mariana T. M. Lima1', Taynara C. Maruyama¹, Isis D. D. Custódio', Eduarda da C. Marinho', Isabela B. Ferreira', Carlos E. Paiva', Yara C. de P. Maia'

${ }^{1}$ Universidade Federal de Uberlândia - Uberlândia (MG), Brazil.

The aim of this prospective study was to investigate the association between eating frequency, diet quality, and nutritional status of 55 women diagnosed with breast cancer, followed up on the three times of chemotherapy, beginning (T0), during (T1), and after the end of treatment (T2). Dietary data were obtained by applying nine nonconsecutive 24h dietary recalls (24HR) and the Brazilian Healthy Eating Index Revised (BHEI-R) was used for qualitative analysis of diet. The average of the eating frequency was established by summing the number of daily eating episodes in the three 24HR of each time, considering at least $30 \mathrm{kcal}(125.52 \mathrm{~kJ})$ per episode. Anthropometric variables, such as, Waist Circumference, Waist-toHeight Ratio, Waist-Hip Ratio, and Body Mass Index were obtained in all the three times. Women who reported higher daily eating frequency [above the median value of each time (T0 and T1: 4.67; T2: 4.33 eating episodes)] presented better anthropometric parameters, in T0 and T1, as well as higher scores for specific components, and BHEI-R global score in T1 and T2. In generalized linear models (GLzM), the daily eating frequency was negatively associated with all the anthropometric variables in $\mathrm{T} 0$ and, specifically, with the Waist-to-Height Ratio in T1. There were also positive associations for the following four BHEI-R components in the three times: Total Fruits; Whole Fruits (excluding fruit juices); Vegetables Dark Green, and Orange Vegetables and Legume. Additionally, in T1 and T2 the daily eating frequency was positively associated with the total BHEI-R score, and also with the Whole Grains in T1. The results of this study suggest that women with breast cancer who have higher daily eating frequency present better nutritional status at the beginning of chemotherapy and better quality of diet for different food components during and at the end of the treatment. 